\title{
Cushing's Disease Associated With Unruptured Large Internal Carotid Artery Aneurysm -Case Report-
}

\author{
Yasuo Nishijima, Yoshikazu OGAWA*, Kenichi SATO*,**, \\ Yasushi MATSUMOTO**, and Teiji TOMINAGA \\ Department of Neurosurgery, Tohoku University Graduate School of Medicine, \\ Sendai, Miyagi; Departments of * Neurosurgery and \\ ${ }^{* *}$ Neuroendovascular Treatment, Kohnan Hospital, Sendai, Miyagi
}

\begin{abstract}
A 40-year-old woman with Cushing's disease presented with hypertensive cerebral hemorrhage. Neuroimaging detected an unruptured large intracavernous aneurysm, which projected beyond the midline, and thin crescent-shaped adenoma along the aneurysm wall. The aneurysm was treated with endovascular tight packing with coils. Transsphenoidal adenomectomy was then safely performed. The signs of Cushing's disease were resolved, and she was discharged without deficits. The first line therapy for Cushing's disease is transsphenoidal adenomectomy. However, the therapeutic strategy and optimal timing of treatment are unclear for Cushing's disease with large intracavernous aneurysm. The present case shows that transsphenoidal surgery was safely possible with minimal invasiveness after embolization of the intracavernous aneurysm.
\end{abstract}

Key words: Cushing's disease, embolization, internal carotid artery aneurysm, pituitary, transsphenoidal surgery

\section{Introduction}

Cushing's disease is a pathologically benign but clinically serious and life threatening disorder because of the associated risks of infection, glucose intolerance, and hypertension. Untreated Cushing's disease quadruples the normal mortality rate, and the 5-year survival rate is around $50 \%{ }^{7}{ }^{7}$ The recommended treatment for Cushing's disease is transsphenoidal adenomectomy, ${ }^{3)}$ which achieves remission in $70-90 \%$ of cases treated by skillful specialized surgeons. ${ }^{6)}$ Cushing's disease associated with large intracavernous aneurysm is very rare, and the optimal therapeutic strategy and timing are unclear. ${ }^{14)}$

We treated a patient with Cushing's disease, who initially presented with hypertensive cerebral hemorrhage with an unruptured large intracavernous aneurysm projecting beyond the midline. Transsphenoidal surgery was safely performed after endovascular embolization of the aneurysm.

\section{Case Report}

A 40-year-old woman was admitted to Kohnan Hospital suffering from severe headache and right hemiparesis in April 2009. Blood pressure was $190 / 100 \mathrm{mmHg}$. She was slightly confused with disorientation, and had right

Received November 10, 2009;

Accepted January 15, 2010 hemiparesis. Computed tomography revealed left putaminal hemorrhage. Digital subtraction angiography (DSA) showed a large internal cerebral artery (ICA) aneurysm in the right cavernous segment, which was thought to have no relationship to the hemorrhage (Fig. 1). Conservative treatment with antihypertensive therapy was begun. The aneurysm was clinically asymptomatic, so was only observed. Her hypertension remained intractable a few days later, and re-examination of her physical status disclosed moon face and central obesity, so Cushing's syndrome
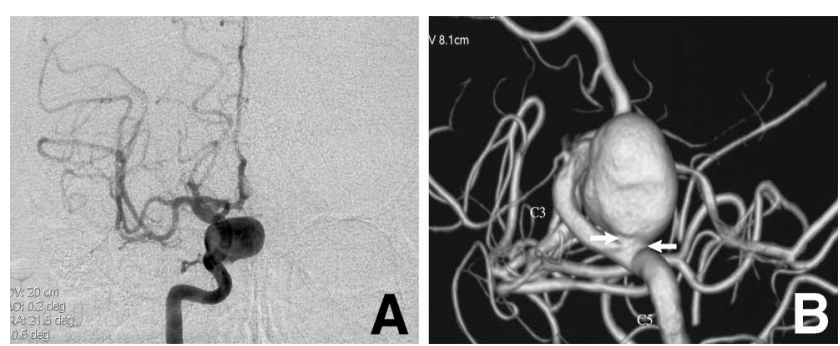

Fig. 1 Digital subtraction angiogram (A) showing a large aneurysm in the cavernous segment of the internal carotid artery (ICA), which projected to the opposite side beyond the midline. Three-dimensional angiogram (B) showing a large aneurysm originating from the horizontal segment of the right ICA, with maximum diameter of $18.3 \mathrm{~mm}$. Arrows indicate the comparatively narrow orifice of the aneurysm. 
was suspected.

Serum basal levels of adrenocorticotropic hormone (ACTH) and cortisol were elevated to $77.6 \mathrm{pg} / \mathrm{ml}$ and 51.5 $\mu \mathrm{g} / \mathrm{dl}$, respectively, and the circadian secretion rhythm had disappeared (Fig. 2). The corticotropin-releasing hormone (CRH) loading test showed normal response, and serum cortisol level was suppressed with both $1 \mathrm{mg}$ and 8 mg of dexamethasone. Magnetic resonance (MR) imaging showed a large aneurysm projecting from the right to the opposite side over the midline. A thin crescent-shaped less enhanced area was shown between the surface of the aneurysm wall and normal pituitary gland indicating pituitary adenoma (Fig. 3). Selective venous sampling of ACTH was performed. Since cannulation into the cavernous sinus was thought extremely hazardous, sampling was performed without the points in the cavernous sinus, and significant step up was shown (right inferior petrosal

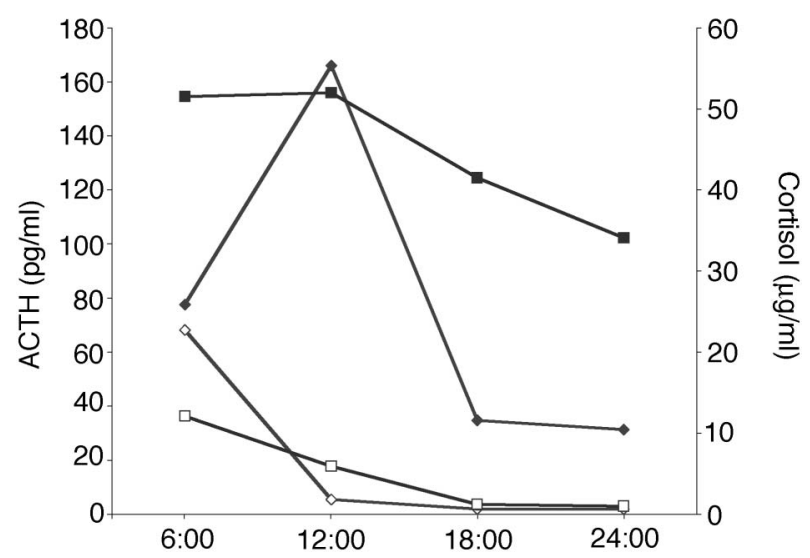

Fig. 2 Circadian levels of adrenocorticotropic hormone (ACTH) and cortisol. Closed symbol lines indicating preoperative serum levels of ACTH $(\diamond)$ and cortisol $(\bullet)$ were elevated, and circadian secretion rhythm had disappeared. Open symbol lines indicating postoperative serum values of ACTH $(\diamond)$ and cortisol $(\square)$ fell into the normal range, and circadian rhythm of hormone secretion had recovered.

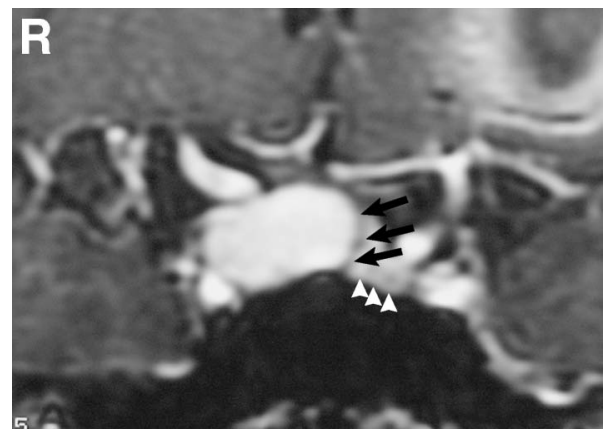

Fig. 3 Dynamic coronal magnetic resonance image with contrast medium revealing the large aneurysm in the right sellar lesion, projecting to the opposite side over the midline, and a less enhanced crescent-shaped adenoma (arrows) between the aneurysm surface and normal pituitary gland (arrowheads). vein [IPS]/internal jugular vein $[$ IJV] $=6740 / 52.3 \mathrm{pg} / \mathrm{ml}$, left IPS/IJV = 1900/51.2 pg/ml). The CRH loading test during venous sampling showed 27-fold excess response of ACTH at the right IPS (pre-loading/post-loading = $6740 / 187000 \mathrm{pg} / \mathrm{ml}$ ). Therefore, the diagnosis was Cushing's disease. However, the ACTH value may have been influenced by cross-flow in the cavernous sinus, so local laterality was not evaluated by this venous sampling.

Endovascular embolization of the aneurysm was planned as a precaution for transsphenoidal surgery in June 2009. Her neurological status had improved and she was independent in life under medication with three types of antihypertensive agent. The aneurysm was tightly packed with Guglielmi detachable coils (GDCs) (GDC18; Target Therapeutics-Boston Scientific, Fremont, Calif., U.S.A.), TruFill-Orbit standard coils (Cordis, Johnson and Johnson, Miami, Fla., U.S.A.), Microsphere coils (Micrus Endovascular Corporation, San Jose, Calif., U.S.A.), and ED10 extrasoft coils (Kaneka Medix Corporation, Kanagawa) to provide the best tolerance to mechanical stress at the aneurysm wall. DSA showed complete obliteration of the aneurysm, and 3.0 T MR imaging showed no blood flow into the aneurysm (Fig. 4).

Absence of blood flow into the aneurysm was repeatedly confirmed, and then transsphenoidal adenomectomy was performed 14 days after embolization. Longitudinal dissection of the normal pituitary gland was performed at the left lateral wing of sella turcica, followed by careful latitudinal dissection to the right side, disclosing the large
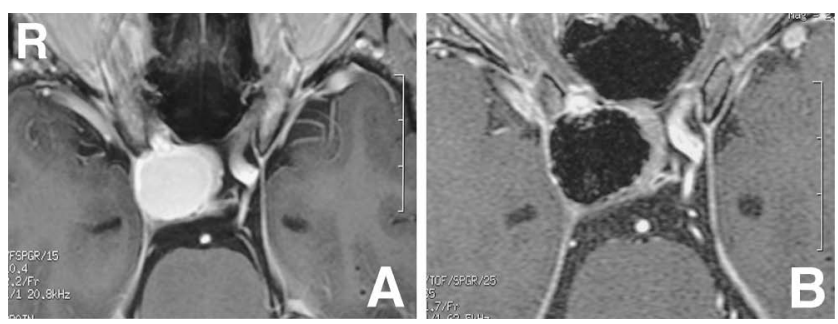

Fig. 4 Pre- (A) and postoperative (B) axial $\mathrm{T}_{1}$-weighted magnetic resonance images with contrast medium showing no blood flow into the aneurysm.

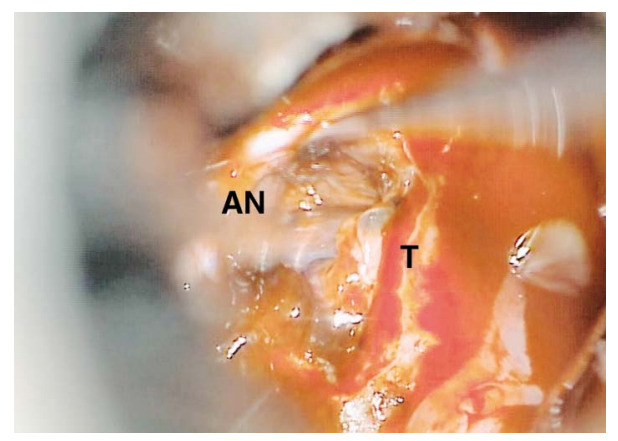

Fig. 5 Intraoperative photograph showing the thrombosed aneurysm (AN) and a small amount of white tumor (T) at the aneurysm surface. 
black thrombosed aneurysm. The aneurysm wall was thinned and partly transparent, and no blood flow or pulsation were observed in the aneurysm. A small amount of white tumor was found by extremely careful exploration along the aneurysm wall, and was easily dissected from normal pituitary gland (Fig. 5). No aneurysmal bleeding occurred throughout the operation.

Slight diplopia and mydriasis in the right eye developed on postoperative day 1, but disappeared in a few days. Endocrinological examinations performed on 15 days after the operation showed that the basal values of ACTH and cortisol were within the normal ranges and circadian secretion rhythm had recovered (Fig. 2). The CRH loading test showed the basal value of ACTH was $21.0 \mathrm{pg} / \mathrm{ml}$ and the peak value was $21.7 \mathrm{pg} / \mathrm{ml}$, and dexamethasone suppression tests also showed normal responses. She was discharged on foot with no neurological deficits on postoperative day 16. Two months later she had lost $7.5 \mathrm{~kg}$ of body weight and antihypertensive medication was reduced from 3 to 2 types. She has been free of relapse, and careful follow up has been continued.

\section{Discussion}

Cavernous sinus aneurysms originating from the anterior genu of the ICA should be surgically treated because of the risk of subarachnoidal hemorrhage, but other types of cavernous aneurysms are considered to have more benign clinical courses. Simple observation is generally recommended in the absence of severe cranial nerve symptoms such as intolerable ophthalmalgia or progressive visual disturbance. However, severe nasal bleeding or carotid cavernous fistula have been reported, so the indications for surgery of this type of aneurysm are not fully established. ${ }^{8-10)}$ In our case, the aneurysm was asymptomatic and originated from the horizontal segment of the ICA, so no intervention was required. However, change of treatment strategy had become essential because of the complication with Cushing's disease. Coexistence of cerebral aneurysms and pituitary adenomas was reported in $7.4 \%$ of cases in past series, ${ }^{4)}$ and tended to coexist with growth hormone producing adenomas. ${ }^{15)}$ However, the mechanism and relationship between Cushing's disease and cerebral aneurysm has still not been elucidated.

Most cases of large intracavernous aneurysms have been treated by occlusion of the ICA or combined occlusion and bypass. Only a few patients were treated by direct clipping. ${ }^{2)}$ Recently, endovascular trapping of the ICA and bypass surgery have been reported.11) Transsphenoidal surgery would be safer after ICA occlusion, but delayed brain infarction after proximal ligation of the ICA ${ }^{13)}$ and formation of flow-related aneurysms may occur. ${ }^{16)}$ Careful evaluation of the long-term prognosis should be made after ICA occlusion. In our case, the neck of the aneurysm was comparatively narrow (dome/neck ratio 2.9), so endovascular embolization was planned. Tight packing of the aneurysm was necessary to tolerate the intense mechanical stimuli during surgery. Finally, complete obliteration of the aneurysm using thick coils (GDC18) for framing and soft coils (ED10 extrasoft) was achieved to ex- clude any neck remnant. 3.0 T MR imaging was performed to assess intraaneurysm blood flow on postembolization days 1 and 7 , and complete obliteration was confirmed (Fig. 4). Organization of the aneurysm cavity ${ }^{1)}$ and new formation of endothelial cells can be expected within 2 weeks, ${ }^{5,12)}$ so transsphenoidal surgery was performed on postembolization day 14 . The operative findings confirmed completely organized aneurysm, which could tolerate mechanical stress, and removal of the adenoma was safely performed.

The present case indicates that the hemostatic effect of coil-embolized aneurysms is resistant to the mechanical stress associated with transsphenoidal selective adenomectomy.

\section{References}

1) Bavinzski G, Richling B, Binder BR, Gruber A, Talazoglu V, Dietrich W, Schwendtenwein I, Plenk H Jr: Histopathological findings in experimental aneurysms embolized with conventional and thrombogenic/antithrombolytic Guglielmi coils. Minim Invasive Neurosurg 42: 167-174, 1999

2) Date I, Tokunaga K, Sugui K: [Multimodality therapy for large and giant intracavernous internal carotid artery aneurysms]. Surgery for Cerebral Stroke 36: 12-18, 2008 (Japanese)

3) De Martin M, Pecori Giraldi F, Cavagnini F: Cushing's disease. Pituitary 9: 279-287, 2006

4) Hatam A, Greitz T: Ectasia of cerebral arteries in acromegaly. Acta Radiol Diagn (Stockh) 12: 410-418, 1972

5) Hino K, Konishi Y, Shimada A, Kurita H, Sato E, Hara M, Shiokawa Y, Saito I: Morphologic changes in neo-intimal proliferation in an experimental aneurysm after coil embolization: effect of factor XIII administration. Neuroradiology 46: 996-1005, 2004

6) Hofmann BM, Hlavac M, Martinez R, Buchfelder M, Müller OA, Fahlbusch R: Long-term results after microsurgery for Cushing disease: experience with 426 primary operations over 35 years. J Neurosurg 108: 9-18, 2008

7) Kelly DF: Transsphenoidal surgery for Cushing's disease: a review of success rates, remission predictors, management of failed surgery, and Nelson's Syndrome. Neurosurg Focus 23(3): E5, 2007

8) Kupersmith MJ, Hurst R, Berenstein A, Choi IS, Jafar J, Ransohoff J: The benign course of cavernous carotid artery aneurysms. J Neurosurg 77: 690-693. 1992

9) Linskey ME, Sekhar LN: Which cavernous sinus aneurysms should be treated? J Neurosurg 78: 1008-1009, 1993

10) Linskey ME, Sekhar LN, Hirsh WL Jr, Yonas H, Horton JA: Aneurysms of the intracavernous carotid artery: natural history and indications for treatment. Neurosurgery 26: 933-938, 1990

11) Liu JK, Fleseriu M, Delashaw JB Jr, Ciric IS, Couldwall WT: Treatment options for Cushing's disease after unsuccessful transsphenoidal surgery. Neurosurg Focus 23(3): E8, 2007

12) Raymond J, Guilbert F, Metcalfe A, Gévry G, Salazkin I, Robledo O: Role of the endothelial lining in recurrences after coil embolization: prevention of recanalization by endothelial denudation. Stroke 35: 1471-1475, 2004

13) Roski RA, Spetzler RF, Nulsem FE: Late complications of carotid ligation in the treatment of intracranial aneurysms. J Neurosurg 54: 583-587, 1981

14) Sade B, Mohr G, Tampieri D, Rizzo A: Intrasellar aneurysm and a growth hormone-secreting pituitary macroadenoma. 
Case report. J Neurosurg 100: 557-559, 2004

15) Wakai S, Fukushima T, Furihata $T$, Sano K: Association of cerebral aneurysm with pituitary adenoma. Surg Neurol 12: 503-507, 1979

16) Winn HR, Richardson AE, Jane JA: Late morbidity and mortality of common carotid ligation for posterior communicating aneurysms. A comparison to conservative treatment. J
Neurosurg 47: 727-736, 1997

Address reprint requests to: Yoshikazu Ogawa, M.D., Ph.D., Department Neurosurgery, Kohnan Hospital, 4-20-1 Nagamachi-Minami, Taihaku-ku, Sendai 982-0012, Japan. e-mail: yogawa@kohnan-sendai.or.jp 